\title{
LYAPUNOV STABILITY THEORY FOR DYNAMIC SYSTEMS ON TIME SCALES ${ }^{1}$
}

\author{
BILLUR KAYMAKÇALAN \\ Florida Institute of Technology \\ Department of Applied Mathematics \\ Melbourne, Florida 32901-6988 U.S.A.
}

\begin{abstract}
By use of the necessary calculus and the fundamental existence theory for dynamic systems on time scales, in this paper, we develop Lyapunov's second method in the framework of general comparison principle so that one can cover and include several stability results for both types of equations at the same time.
\end{abstract}

scales.

Key words: Lyapunov stability, dynamic systems on time

AMS (MOS) subject classifications: $\quad 34 \mathrm{D} 20$.

\section{INTRODUCTION}

As is well known $[4,5,6]$, one can develop qualitative behavior of differential systems as well as difference equations by employing Lyapunov-like functions and the theory of corresponding inequalities. We also realize in this process, that several results of differential equations do just translate themselves into analogous results in difference equations. This naturally raises the question whether it is possible to unify the theory of differential and difference equations into a single set up. The answer is yes and we now have necessary calculus and the fundamental existence theory for dynamic systems on times scales $[1,2,3]$.

In this paper, we shall develop Lyapunov's second method in the framework of general comparison principle so that one can cover and include several stability results for both types of equations at the same time.

\footnotetext{
${ }^{1}$ Received: March, 1992. Revised: July, 1992.
} 


\section{COMPARISON PRINCIPLE}

Let $\mathbb{T}$ be a time scale (any closed subset of $\boldsymbol{R}$ with order and topological structure in a canonical way) with $t_{0} \geq 0$ as a minimal element. By an interval, we always mean in the sequel the intersection of a real intervale with the given time scale. If a time sclae has a maximal element which is also left-scattered, it is called a degenerate point. Let $\mathrm{T}^{k}$ repesent the set of all non-degenerate points of $T$. We refer to [1,2] for further definitions on time scales.

Let us begin with the following definition.

Definition 2.1: The mapping $f: T \times \mathbb{R}^{n} \rightarrow \mathbb{R}^{n}$ is said to be $r d$-continuous and is denoted by $f \in C_{r d}\left[\mathbb{T} \times \mathbb{R}^{n}, \mathbb{R}^{n}\right]$ if

(i) it is continuous at each $(t, x)$ with right-dense or maximal $t$ and

(ii) the limits $f\left(t^{-}, x\right)=\lim _{(s, y) \rightarrow\left(t^{-}, x\right)} f(s, y)$ and $\lim _{y \rightarrow x} f(t, y)$ exist at each $(t, x)$ with left-dense $t$.

We refer to $[1,3]$ for further definitions regarding time scales.

Consider the dynamic system

$$
x^{\Delta}=f(t, x), t \in \mathbb{T}, x\left(t_{0}\right)=x_{0}, t_{0} \geq 0,
$$

where $f \in C_{r d}\left[\mathbb{T} \times \mathbb{R}^{n}, \mathbb{R}^{n}\right]$ and $x^{\Delta}$ denotes the derivative of $x$ with respect to $t \in \mathbb{T}$. Assume, for convenience, that the solutions $x(t)=x\left(t, t_{0}, x_{0}\right)$ of $(2.1)$ exist and are unique for $t \geq t_{0}$ and $f(t, 0)=0$ so that we have the trivial solution $x=0$. For local and global existence results for (2.1) see [3].

Definition 2.2: Let $V \in C_{r d}\left[\mathbb{T}^{k} \times \mathbb{R}^{n}, \mathbb{R}_{+}\right]$and $\mu^{*}(t)$ be as in Definition 2.2 in [3]. Then we define

$$
D_{-} V^{\Delta}(t, x) \equiv \lim _{\mu^{*}(t) \rightarrow 0} \inf \frac{V(t, x)-V\left(t-\mu^{*}(t), x-\mu^{*}(t) f(t, x)\right)}{\mu^{*}(t)}
$$

and

$$
D^{+} V^{\Delta}(t, x) \equiv \lim _{\mu^{*}(t) \rightarrow 0} \sup \frac{V\left(t+\mu^{*}(t), x+\mu^{*}(t) f(t, x)\right)-V(t, x)}{\mu^{*}(t)}
$$

If $V$ is differentiable, then $D_{-} V^{\Delta}(t, x)=D^{+} V^{\Delta}(t, x)=V^{\Delta}(t, x)$ where $V^{\Delta}(t, x)=$ $V_{t}^{\Delta}(t, x)+V_{x}^{\Delta}(t, x) f(t, x)$. Here $V_{t}^{\Delta}$ is considered as in Definition 2.5 in [3] and $V_{x}^{\Delta}$ is taken as the normal derivative. 
Having the comparison theorem (see Theorem 5.2 in [3]) at our disposal, we can prove the required comparison result in terms of Lyapunov-like functions.

Theorem 2.1: $\quad$ Let $V \in C_{r d}\left[\mathbb{T} \times \mathbb{R}^{n}, \mathbb{R}_{+}\right]$and $V(t, x)$ be locally Lipschitzian in $x$ for each $t$. Assume that

$$
D_{-} V^{\Delta}(t, x) \leq g(t, V(t, x)),(t, x) \in \mathbb{T} \times \mathbb{R}^{n}
$$

where $g \in C_{r d}\left[\mathbb{T} \times \mathbb{R}_{+}, \mathbb{R}\right]$ and $g(t, u) \mu^{*}(t)$ be non-decreasing in $u$ for each $t \in \mathbb{T}^{k}$. Let $r(t)=r\left(t, t_{0}, u_{0}\right)$ be the maximal solution of the scalar differential equation

$$
u^{\Delta}=g(t, u), u\left(t_{0}\right)=\left(u_{0}\right) \geq 0
$$

existing to the right of $t_{0}$. If $x(t)=x\left(t, t_{0}, u_{0}\right)$ is any solution of $(2.1)$ such that

$$
V\left(t_{0}, x_{0}\right) \leq u_{0}
$$

then

$$
V(t, x(t)) \leq r(t), t \in \mathrm{T}
$$

Proof: Let $x(t)$ be any solution of (2.1) defined for $t \geq t_{0}$ such that (2.5) holds. Define

$$
m(t)=V(t, x(t))
$$

so that $m\left(t_{0}\right) \leq u_{0}$. Then,

$$
\begin{gathered}
m(t)-m\left(t-\mu^{*}(t)\right)=V(t, x(t))-V\left(t-\mu^{*}(t), x(t)-\mu^{*}(t) f(t, x(t))-\epsilon \mu^{*}(t)\right) \\
=V(t, x(t))-V\left(t-\mu^{*}(t), x-\mu^{*}(t) f(t, x)\right)+V\left(t-\mu^{*}(t), x-\mu^{*}(t) f(t, x)\right) \\
-V\left(t-\mu^{*}(t), x(t)-\mu^{*}(t) f(t, x(t))-\epsilon \mu^{*}(t)\right)
\end{gathered}
$$

Since $V(t, x)$ is locally Lipschitzian in $x$ for each $t$ and $\frac{\epsilon\left(\mu^{*}(t)\right)}{\mu^{*}(t)} \rightarrow 0$ as $\mu^{*}(t) \rightarrow 0$, we obtain, in view of $(2.4)$, the relation

$$
D_{-} m^{\Delta}(t) \leq D_{-} V^{\Delta}(t, x(t)) \leq g(t, m(t)), t \in \mathbb{T},
$$

where $D_{-} m^{\Delta}(t)=\lim _{\mu^{*}(t) \rightarrow 0} i n f \frac{m(t)-m\left(t-\mu^{*}(t)\right)}{\mu^{*}(t)}$ and noting that Theorem 5.2 in [3] remains valid when $m^{\Delta}(t)$ is replaced by $D_{-} m^{\Delta}(t)$. Hence Theorem 5.2 in [3] yields the estimate (2.6). The proof is complete. 
Corollary 2.1: The function $g(t, u) \equiv 0$ is admissible in Theorem 2.1 to yield

$$
V(t, x(t)) \leq V\left(t_{0}, x_{0}\right), t \in \mathrm{T}
$$

\section{STABILITY CRITERIA}

Let us begin by proving the following general result which offers stability criteria for differential systems and difference equations at the same time.

Theorem 3.1: $\quad$ Assume that

(i) $\quad V \in C_{r d}\left[\mathbf{T} \times \mathbb{R}^{n}, \mathbb{R}_{+}\right], V(t, x)$ is locally Lipschitzian in $x$ for all $t \in \mathbb{T}$;

(ii) $\quad b(\|x\|) \leq V(t, x) \leq a(\|x\|)$ for $(t, x) \in \mathbf{T} \times \mathbb{R}^{n}$ where $a, b \in \mathscr{G}=[\sigma \in C$ $\left[\mathbb{R}_{+}, \mathbb{R}_{+}\right]: \sigma(0)=0$ and $\sigma(u)$ is increasing in $\left.u\right]$;

(iii) $g \in C_{r d}\left[\mathbb{T} \times \mathbb{R}_{+}, \mathbb{R}\right]$ and

$$
D_{-} V^{\Delta}(t, x) \leq g(t, V(t, x)),(t, x) \in \mathbb{T} \times \mathbb{R}^{n} .
$$

Then the stability properties of the trivial solution of

$$
u^{\Delta}=g(t, u), u\left(t_{0}\right)=u_{0} \geq 0
$$

imply the corresponding stability properties of the trivial solution of (2.1).

Proof: Let $\epsilon>0$ and $t_{0} \in \mathbb{T}$ be given. Suppose that the trivial solution of (3.1) is stable. Then given $b(\epsilon)>0$ and $t_{0} \in \mathrm{T}$, there exists a $\delta_{1}=\delta_{1}\left(t_{0}, \epsilon\right)>0$ such that

$$
u_{0}<\delta_{1} \Rightarrow u(t)<b(\epsilon), t \in \mathbb{T}
$$

where $u(t)=u\left(t, t_{0}, u_{0}\right)$ is any solution of (3.1). Choose $\delta=\delta\left(t_{0}, \epsilon\right)>0$ such that

$$
a(\delta)<\delta_{1} .
$$

We claim that if $\left|x_{0}\right|<\delta$, then $|x(t)|<\epsilon, t \in \mathbf{T}$, where $x(t)=x\left(t, t_{0}, x_{0}\right)$ is any solution of (2.1). If this is not true, there would exist a $t_{1} \in \mathbf{T}, t_{1}>t_{0}$ and a solution $x(t)=x\left(t, t_{0}, x_{0}\right)$ of (2.1) satisfying

$$
|x(t)|<\epsilon, t_{0} \leq t<t_{1} \text { and }\left|x\left(t_{1}\right)\right| \geq \epsilon .
$$

Setting $m(t)=V(t, x(t))$ for $t_{0} \leq t \leq t_{1}$ and using condition (iii), we get by Theorem 2.1 , the estimate

$$
V(t, x(t)) \leq r\left(t, t_{0}, u_{0}\right), t_{0} \leq t \leq t_{1}
$$


where $r\left(t, t_{0}, u_{0}\right)$ is the maximal solution of (3.1) with $V\left(t_{0}, x_{0}\right) \leq u_{0}$. Now the relations (3.2), (3.4), (3.5) and the assumption (ii) yield

$$
b(\epsilon) \leq b\left(\left|x\left(t_{1}\right)\right|\right) \leq V\left(t, x\left(t_{1}\right)\right) \leq r\left(t_{1}, t_{0}, u_{0}\right)<b(\epsilon),
$$

since $u_{0}=V\left(t_{0}, x_{0}\right) \leq\left(\left|x_{0}\right|\right)<a(\delta)<\delta_{1}$ by (3.3). This contradiction proves the claim.

Other stability properties may be proved in a similar manner and hence the proof is complete.

Remark: Usually when stability properties for differential systems are proved, one imposes conditions on $V(t, x)$ only in $\mathbb{R}_{+} \times S(\rho)$, where $S(\rho)=\left[x \in \mathbb{R}^{n}:|x|<\rho\right]$, because stability notions are of local nature relative to the trivial solution. On the other hand, when we deal with difference equations, we need, either to assume $S(\rho)$ is invariant or work in the entire $\mathbb{R}^{n}$, since we have no control of how large the solutions grow being discontinuous. To avoid assuming the invariance of $S(\rho)$ for some $\rho>0$, we have imposed conditions everywhere. In the present set up, all we need to concentrate is on the estimate (3.4). If $t_{1}$ is a nonscattered point, that is, say left dense, we then get $\left|x\left(t_{1}\right)\right|=\epsilon$ and if $t_{1}$ is scattered point, $\left|x\left(t_{1}\right)\right| \geq \epsilon$ and it may happen that $\left|x\left(t_{1}\right)\right|>\rho$ if we work in $S(\rho)$.

We can obtain from Theorem 3.1, Lyapunov's theorems for continuous and discrete cases, immediately. This we state as corollaries.

\section{Corollary 3.1:}

(i) The function $g(t, u) \equiv 0$ is admissible in Theorem 3.1 to yield uniform stability of the zero solution of $(2.1)$.

(ii) The function $g(t, u)=-c_{0}(u)$, where $c_{0} \in 96$, is admissible to imply uniform asymptotic stability of the trivial solution of (2.1).

Usually Lyapunov's theorem on uniform asymptotic stability should have the assumption $V^{\Delta}(t, x) \leq-c(|x|)$. However, it is easy to see that if $V$ has an upper estimate as in (ii) of Theorem 3.1, one can obtain the assumption of Corollary 3.1, (ii).

As an example, consider the linear system

$$
x^{\Delta}=A x, x(0)=x_{0}
$$

with $t_{0}=0$, where $A$ is an $n \times n$ matrix. As usual, we choose

$$
V(t, x)=x^{T} P x
$$

where $P$ is an $n \times n$ symmetric matrix that is positive definite. Then 
$V^{\Delta}(t, x)=x^{T}\left[A^{T} P+P A\right] x$. If the Lyapunov equation

$$
A^{T} P+P A+Q=0
$$

is satisfied for a positive definite $n \times n$ matrix $Q$, then it is clear that $V^{\Delta}(t, x)$ is negative definite and therefore, the trivial solution of (3.6) is uniformly asymptotically stable by Corollary 3.1 .

We shall next consider the case when $V(t, x)$ is not decrescent, that is, $V(t, 0) \equiv 0$ and extend Marachkov's result in the present set up. Since Salvadori's [5] generalization of Marachkov's theorem for differential equations exist, we shall consider the extension in that generality.

Theorem 3.2: $\quad$ Let the assumptions (i) and (ii) of Theorem 3.1 hold for $V$. Further assume for $W \in C_{r d}\left[\mathbb{T} \times \mathbb{R}^{n}, \mathbb{R}_{+}\right]$that $W(t, x)$ is locally Lipschitzian in $x$, it is positive definite, $D^{+} W^{\Delta}(t, x)$ is bounded from above or from below for $(t, x) \in \mathbb{T} \times \mathbb{R}^{n}$,

$$
D^{+} V^{\Delta}(t, x) \leq-c(W(t, x)), c \in 96
$$

Then, the system (2.1) is asymptotically stable.

Proof: Suppose that $\left\|x_{0}\right\|<\delta_{0}$. The stability of the zero solution of (2.1) follows from Corollary 3.1 since $D^{+} V^{\Delta}(t, x) \leq-c(W(t, x))$ readily implies $D^{+} V^{\Delta}(t, x) \leq 0$. Since $W(t, x)$ is positive definite, it is enough to prove that $\lim _{t \rightarrow \infty} W(t, x(t))=0$ for any solution $x(t)$ of (2.1). We first note that $\lim _{t \rightarrow \infty} \inf W(t, x(t))=0$, for otherwise in view of (3.9) we get $V(t, x(t)) \rightarrow-\infty$ as $t \rightarrow \infty$.

Suppose that $\lim _{t \rightarrow \infty} \sup W(t, x(t)) \neq 0$. Then for any $\epsilon>0$, there exist divergent sequences $\left\{t_{n}\right\},\left\{t_{n}^{*}\right\}$ such that $t_{i}<t_{i}^{*}<t_{i+1}, i=1,2, \ldots$ and one of the following holds:

(i) $\quad t_{i}$ is right dense, $t_{i}^{*}$ is left dense

(ii) $t_{i}$ is left dense, $t_{i}^{*}$ is right dense

(iii) $t_{i}$ is right or left dense, $t_{i}^{*}$ is right or left scattered

(iv) $t_{i}$ is right or left scattered, $t_{i}^{*}$ is right or left dense,

with

$$
\left\{\begin{array}{cc}
W\left(t_{i}, x\left(t_{i}\right)\right)=\frac{\epsilon}{2}, & W\left(t_{i}^{*}, x\left(t_{i}^{*}\right)\right)=\epsilon \text { and } \\
\frac{\epsilon}{2}<W(t, x(t))<\epsilon, & t \in\left(t_{i}, t_{i}^{*}\right), i=1,2, \ldots .
\end{array}\right.
$$

Of course, we could have, instead of (3.10), given

$$
\left\{\begin{array}{cl}
W\left(t_{i}, x\left(t_{i}\right)\right)=\epsilon, & W\left(t_{i}^{*}, x\left(t_{i}^{*}\right)\right)=\frac{\epsilon}{2} \text { and } \\
\frac{\epsilon}{2}<W(t, x(t))<\epsilon \text { for } & t \in\left(t_{i}, t_{i}^{*}\right), i=1,2, \ldots
\end{array}\right.
$$


Suppose that $D^{+} W^{\Delta}(t, x) \leq M$. Then it is easy to obtain, using (3.10) the relation $t_{i}^{*}-t_{i}>\frac{\epsilon}{2 M}$. In view of the assumptions $(i)$ and $(i i)$ of Theorem $3.1,(3.9)$ and (3.10), we have for large $n$,

$$
\begin{aligned}
0 \leq V\left(t_{n}^{*}, x\left(t_{n}^{*}\right)\right) & \leq V\left(t_{0}, x_{0}\right)+\sum_{1 \leq i \leq n} \int_{t_{i}}^{t_{i}^{*}} D^{+} V^{\Delta}(s, x(s)) d s \\
& \leq V\left(t_{0}, x_{0}\right)-n c\left(\frac{\epsilon}{2}\right) \frac{\epsilon}{2 M}<0
\end{aligned}
$$

which is a contradiction. Thus $W(t, x(t)) \rightarrow 0$ as $t \rightarrow \infty$ and hence $x(t) \rightarrow 0$ as $t \rightarrow \infty$. The case when $D^{+} W^{\Delta}$ is bounded from below can be proved using (3.11) with similar arguments. In the case that $t_{i}$ and $t_{i}^{*}$ are both left or right scattered, we assume $W\left(t_{i}, x\left(t_{i}\right)\right)=\frac{\epsilon}{2}$, $W\left(t_{i}^{*}, x\left(t_{i}^{*}\right)\right)=\epsilon$ or $W\left(t_{i}, x\left(t_{i}\right)\right)=\epsilon, W\left(t_{i}^{*}, x\left(t_{i}^{*}\right)\right)=\frac{\epsilon}{2}$. Then by use of 4.3 of [2] for definition of Cauchy integrals for scattered points, $(3.12)$ becomes

$$
\begin{aligned}
0 \leq V\left(t_{n}^{*}, x\left(t_{n}^{*}\right)\right) \leq & V\left(t_{0}, x_{0}\right)+\sum_{1 \leq i \leq n}\left[D^{+} V\left(t_{i}, x\left(t_{i}\right)\right)+D^{+} V\left(t_{i}^{*}, x\left(t_{i}^{*}\right)\right)\right] \\
& \leq V\left(t_{0}, x_{0}\right)-n 2 c\left(\frac{\epsilon}{2}\right)<0 \text { for large } n .
\end{aligned}
$$

Hence we still have $W(t, x(t)) \rightarrow 0$ as $t \rightarrow \infty$ and the proof is complete.

Note that, in the above set up, the importance played by points of different nature (such as scattered or dense) of a time scale is clearly seen.

\section{REFERENCES}

[1] B. Aulbach and S. Hilger, "Linear Dynamic Processes with Inhomogeneous Time Scales", Nonlinear Dynamics and Quantum Dynamical Systems, Akademie-Verlag, Berlin, 1990.

[2] S. Hilger, "Analysis on measure chains- A unified approach to continuous and discrete calculus", Res. in Mathematics 18, (1990), pp. 18-56.

[3] B. Kaymakçalan, "Existence and comparison results for dynamic systems on time scale», J.M.A.A. (to appear).

[4] V. Lakshmikantham and S. Leela, "Differential and Integral Inequalities", Vol. I, Academic Press, New York, 1969.

[5] V. Lakshmikantham, S. Leela and A.A. Martynyuk, "Stability Analysis for Nonlinear Systems” Marcel Dekker, Inc., New York, 1989.

[6] V. Lakshmikantham and D. Trigiante, "Theory of Difference Equations: Numerical Methods and Applications", Academic Press, Inc., New York, 1988. 


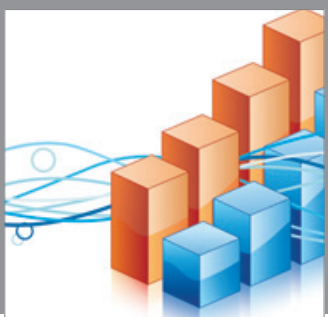

Advances in

Operations Research

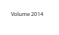

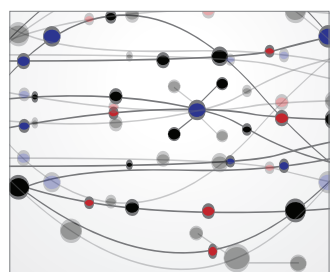

\section{The Scientific} World Journal
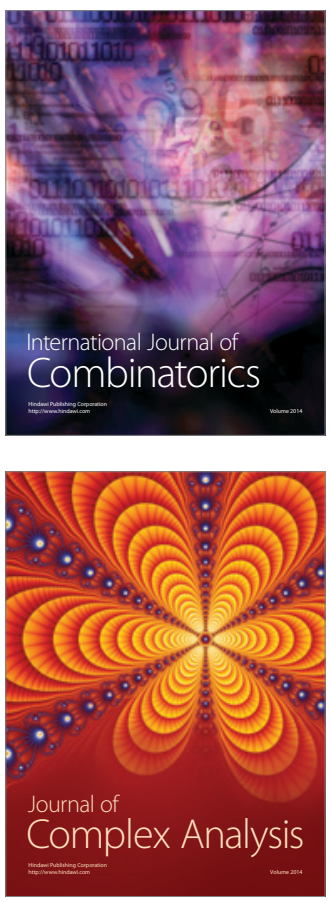

International Journal of

Mathematics and

Mathematical

Sciences
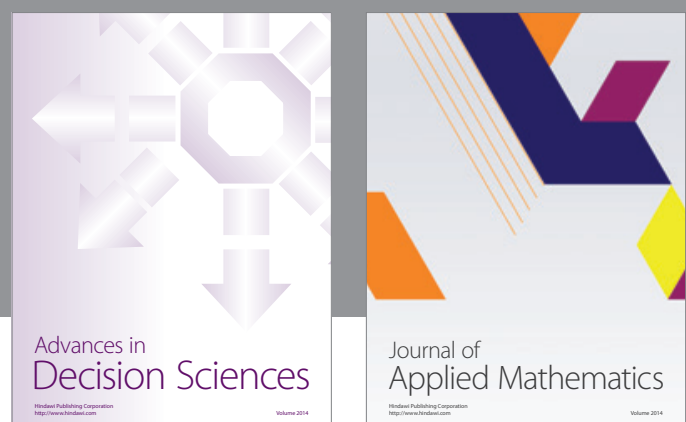

Journal of

Applied Mathematics
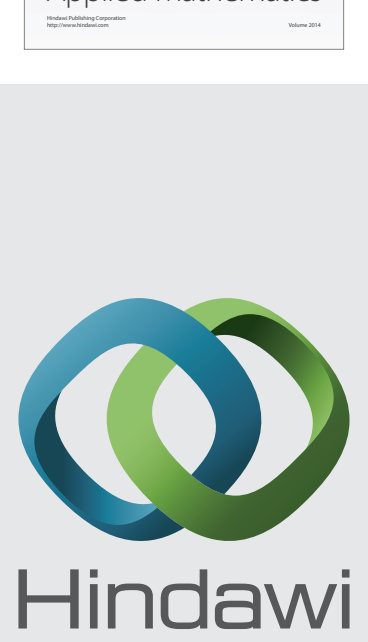

Submit your manuscripts at http://www.hindawi.com
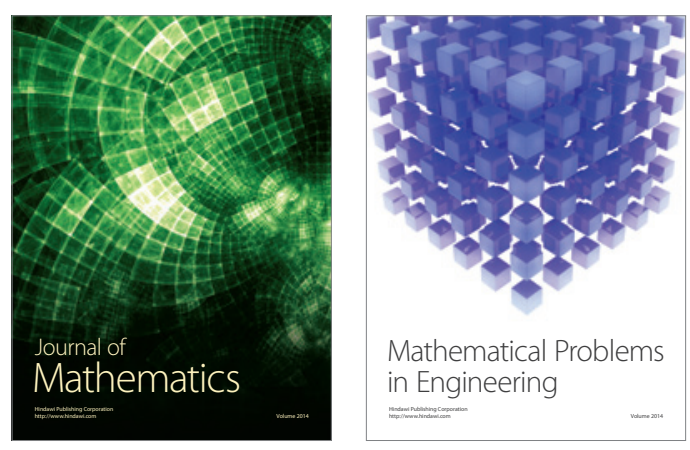

Mathematical Problems in Engineering
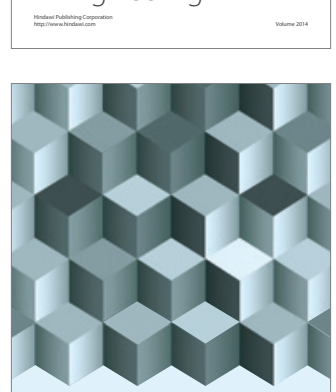

Journal of

Function Spaces
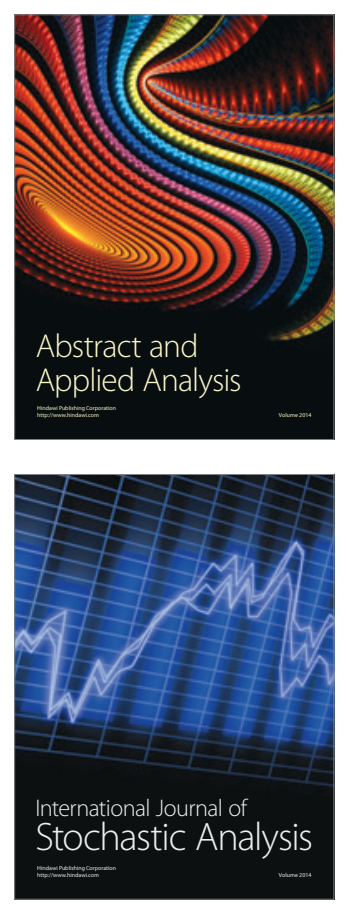

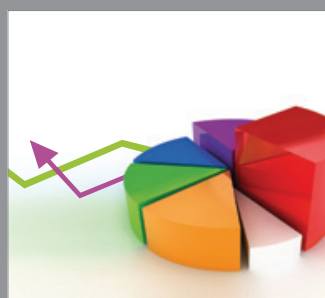

ournal of

Probability and Statistics

Promensencen
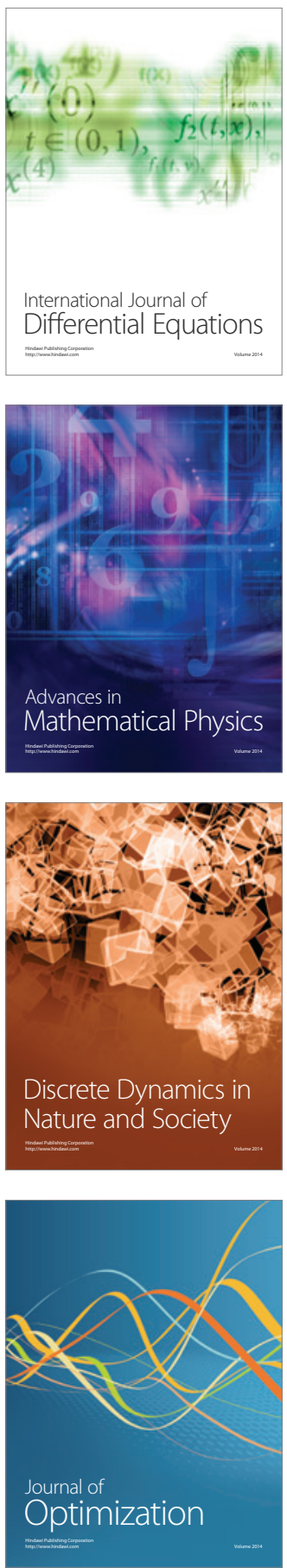